\title{
Population attributable risk estimates of risk factors for contrast-induced acute kidney injury following coronary angiography: a cohort study
}

Li Lei ${ }^{1,2+}$, Yan Xue ${ }^{3 \dagger}$, Zhaodong Guo ${ }^{2 \dagger}$, Bowen Liü ${ }^{4 \dagger}$, Yibo He ${ }^{2 \dagger}$, Feier Song ${ }^{5}$, Jin Liu², Guoli Sun ${ }^{2}$, Liling Chen ${ }^{6}$, Kaihong Chen ${ }^{6}$, Zhiqi Su², Li Pan², Zhidong Huang ${ }^{7}$, Yulu Huang ${ }^{7}$, Xiuqiong Huang ${ }^{7}$, Shiqun Chen², Jiyan Chen ${ }^{1,2^{*}}$ and Yong Liu ${ }^{1,2^{*}}$ (D)

\begin{abstract}
Background: Contrast-induced acute kidney injury (Cl-AKI) is a common complication with poor outcomes following coronary angiography (CAG) or percutaneous coronary intervention (PCI). However, no study has explored the population attributable risks (PARs) of the Cl-AKI risk factors. Therefore, we aimed to identify the independent risk factors of $\mathrm{Cl}$-AKI and estimate their PARs.
\end{abstract}

Methods: We analyzed 3450 consecutive patients undergoing CAG/PCl from a prospective cohort in Guangdong Provincial People's Hospital. Cl-AKI was defined as a serum creatinine elevation $\geq 50 \%$ or $0.3 \mathrm{mg} / \mathrm{dL}$ from baseline within the first 48 to $72 \mathrm{~h}$ after the procedure. Independent risk factors for Cl-AKI were evaluated through stepwise approach and multivariable logistic regression analysis, and those that are potentially modifiable were of interest. PARs of independent risk factors were calculated with their odds ratios and prevalence among our cohort.

Results: The overall incidence of Cl-AKI was 7.19\% $(n=248)$, which was associated with increased long-term mortality. Independent risk factors for Cl-AKI included heart failure (HF) symptoms, hypoalbuminemia, high contrast volume, hypotension, hypertension, chronic kidney disease stages, acute myocardial infarction and age $>75$ years. Among the four risk factors of interest, the PAR of HF symptoms was the highest (38.06\%), followed by hypoalbuminemia (17.69\%), high contrast volume (12.91\%) and hypotension (4.21\%).

Conclusions: These modifiable risk factors (e.g., HF symptoms, hypoalbuminemia) could be important and costeffective targets for prevention and treatment strategies to reduce the risk of $\mathrm{Cl}$-AKI. Intervention studies targeting these risk factors are needed.

Keywords: Catheterization, Acute renal disease, Risk factors, Population attributable risk

\footnotetext{
*Correspondence: chenjiyandr@126.com; liuyong@gdph.org.cn

'Li Lei, Yan Xue, Zhaodong Guo, Bowen Liu and Yibo He contributed equally

to this work.

'The Second School of Clinical Medicine, Southern Medical University,

Guangzhou 510515, Guangdong, China

Full list of author information is available at the end of the article
}

\section{$\triangle B M C$}

C C The Author(s). 2020 Open Access This article is licensed under a Creative Commons Attribution 4.0 International License, which permits use, sharing, adaptation, distribution and reproduction in any medium or format, as long as you give appropriate credit to the original author(s) and the source, provide a link to the Creative Commons licence, and indicate if changes were made. The images or other third party material in this article are included in the article's Creative Commons licence, unless indicated otherwise in a credit line to the material. If material is not included in the article's Creative Commons licence and your intended use is not permitted by statutory regulation or exceeds the permitted use, you will need to obtain permission directly from the copyright holder. To view a copy of this licence, visit http://creativecommons.org/licenses/by/4.0/ The Creative Commons Public Domain Dedication waiver (http://creativecommons.org/publicdomain/zero/1.0/) applies to the data made available in this article, unless otherwise stated in a credit line to the data. 


\section{Background}

Contrast-induced acute kidney injury (CI-AKI) is a common complication of coronary diagnostic and interventional procedures that is significantly associated with the composite endpoint of major adverse renal and cardiovascular events (MARCE) [1-3]. The 2018 European Society of Cardiology (ESC)/European Association for Cardio-Thoracic Surgery (EACTS) Guidelines on myocardial revascularization recommend assessing all patients for the risk of CI-AKI [4]. Screening and identifying patients at risk of CI-AKI would easily and accurately allow prophylactic intervention in those at high risk. Research on the prevention of CI-AKI has focused on the use of intravenous fluids, renal replacement therapies (RRTs), and pharmaceutical agents. One of the challenges in clinical practice is that the population benefits of preventive RRT and drug treatments have not been proved [1, 2]. Clinicians do not have enough information to improve evidence-based screening and prevention efforts.

The population attributable risk (PAR) represents the proportion of disease cases in a population that would not have occurred in the absence of a risk factor [5]. Many observational studies regarding CI-AKI risk factors have been reported, but to our knowledge, no studies quantifying the contributions of risk factors of CI-AKI have been identified [6]. There is a continued need to identify which risk factors have the greatest impact on CI-AKI, especially those that might be potentially modifiable by interventions.

Therefore, we aimed to evaluate the association between several risk factors commonly documented in clinical practice and CI-AKI and to estimate their PARs among a large prospective cohort.

\section{Methods}

\section{Patient selection}

The 3450 consecutive patients undergoing coronary angiography (CAG) or percutaneous coronary intervention (PCI) between January 2010 and October 2012 in Guangdong Provincial People's Hospital were enrolled. The exclusion criteria included contrast exposure within the previous 7 days or 3 days after the procedure, pregnancy, lactation, cardiovascular surgery, no use of lowosmolarity contrast agents, undergoing hemodialysis, missing preoperative or postoperative creatinine, malignancy, and no use of isotonic saline for hydration [7]. The study was approved by the Ethics Committee of Guangdong Provincial People's Hospital. All the patients included in this study signed written informed consent.

\section{Endpoint and definitions}

The primary endpoint of this study was CI-AKI, defined as a serum creatinine (Scr) elevation $\geq 50 \%$ or $0.3 \mathrm{mg} / \mathrm{dL}$ from baseline within the first 48 to $72 \mathrm{~h}$ after the procedure. The secondary endpoint was all-cause death. All eligible patients included were followed up through office visits or telephone interviews at 1 month, 6 months and every year after enrollment until April 2019. High contrast volume was defined as contrast volume $>155$ $\mathrm{mL}$ during the procedure. The cutoff value of " $155 \mathrm{~mL}$ " to determine high contrast volume was derived from the receiver operating characteristic curve that had the maximal sum of sensitivity and specificity [8]. Heart failure (HF) symptoms were defined as New York Heart Association (NYHA) class > I/Killip class > I on presentation. Chronic kidney disease (CKD) was defined as estimated glomerular filtration rate $(\mathrm{eGFR})<60 \mathrm{~mL} / \mathrm{min} / 1.73 \mathrm{~mm}^{2}$. Patients with CKD were also divided into 3 stages (mild: eGFR: $45-60 \mathrm{~mL} / \mathrm{min} / 1.73 \mathrm{~mm}^{2}$; moderate: eGFR: $30-$ $45 \mathrm{~mL} / \mathrm{min} / 1.73 \mathrm{~mm}^{2}$; severe: eGFR: < $30 \mathrm{~mL} / \mathrm{min} / 1.73$ $\mathrm{mm}^{2}$ ) [9]. Hypoalbuminemia was defined as serum albu$\min (\mathrm{ALB})<35 \mathrm{~g} / \mathrm{L}$ [10]. Anemia was defined as hematocrit $<36 \%$ for women and $<39 \%$ for men, and hypotension was defined as systolic blood pressure $<80$ $\mathrm{mmHg}$ for at least $1 \mathrm{~h}$ requiring inotropic support with medications or intra-aortic balloon pump (IABP) within $24 \mathrm{~h}$ peri-procedurally [11]..

\section{Study protocol}

The procedure was performed according to published guidelines [12]. The contrast volume was determined by the operators. A noninvasive treatment strategy was performed according to published guidelines and clinical routines. Scr was measured for all patients at admission and at 1,2 , and 3 days after the procedure.

\section{Statistical analysis}

Risk factors of interest (HF symptoms, hypoalbuminemia, high contrast volume, hypotension) that are potentially modifiable and independently associated with CIAKI were selected based on the result of multivariable logistic regression, previous studies and clinical importance [1].

For continuous variables, data are expressed as mean \pm standard deviation and compared between 2 groups through the independent samples t-test if they were normally distributed; otherwise, data are expressed as median \pm interquartile range and compared between groups using the Wilcoxon rank-sum test. For categorical data (expressed as percentages), Pearson's chi-squared or Fisher's exact test was conducted. Long-term mortality in patients with or without CI-AKI was assessed with Kaplan-Meier survival curves, and equality tests of survival distributions were compared using the log-rank test. Moreover, we did landmark analyses to assess allcause mortality at 90-days and after 90-days. 
Univariable logistic regression was conducted for risk factors that were imbalanced between groups and with missing value $<15 \%$. For variables that had interaction or collinearity between each other, the modifiable or categorical one was preferred for better clinical implication. Factors with significant impotence in univariable logistic regression were then enrolled in a backward stepwise approach which successively removing non-significant covariates $(P>0.05)$ until all the remaining predictors are statistically significant. Multivariable logistic regression model including all the remaining risk factors were then fitted to calculate the odds ratios (ORs) for their impact on CIAKI. PAR was calculated for independent risk factors using the equation $\mathrm{PAR}=\mathrm{P} \quad(\mathrm{OR}-1) /[1+\mathrm{P} \quad(\mathrm{OR}-1)]$, where $\mathrm{P}$ is the prevalence of each risk factor in our database. The standard error of PAR was calculated using the delta method [13]. All data analyses were conducted with $\mathrm{R}$ software (version 3.6.2; $\mathrm{R}$ Foundation for Statistical Computing, Vienna, Austria).

\section{Results}

\section{Baseline characteristics}

All 3450 eligible patients were included in the final analysis, among whom the incidence rate of CI-AKI was 7.19\% $(n=248)$. Table 1 details the patient characteristics. Patients with CI-AKI following CAG were older and emaciated. They had higher proportion of impaired heart and renal function, hypertension, hypoalbuminemia, anemia, acute myocardial infarction (AMI) and coronary artery disease (CAD). Higher heart rate, Creactive protein and serum urea nitrogen were also identified in those complicated with CI-AKI. During their hospitalization, they were more likely to be prescribed diuretics and antibiotics. Higher dose of contrast media during the procedure was also found in those with CIAKI.

\section{The association between $\mathrm{Cl}-\mathrm{AKI}$ and prognosis}

After the procedure, $26(10.48 \%)$ patients who were complicated with CI-AKI underwent hemodialysis, while 8 $(0.25 \%)$ patients without CI-AKI underwent hemodialysis $(P<0.001)$.

During the median follow-up of $7.41(6.21 ; 8.27)$ years, mortality was $17.0 \%(n=586)$ in total, $31.9 \%$ $(n=79)$ in patients with CI-AKI, and $15.8 \%(n=507)$ in patients without CI-AKI $(P<0.001)$. Kaplan-Meier survival curves revealed that patients with CI-AKI following CAG had a higher mortality rate than those without CI-AKI $(\log -\operatorname{rank} P<0.01$; Fig. 1$)$. The significant association between CI-AKI and all cause death at 90-days follow-up was maintained after 90days. (Figure S1).

\section{Risk factors for Cl-AKI}

Multivariable logistic regression revealed that hypoalbuminemia (OR: 1.48, 95\% CI: 1.03-2.13), HF symptoms (OR: 2.12, 95\% CI: 1.46-3.06), high contrast volume (OR: 1.60, 95\% CI: 1.14-2.24), hypotension (OR: 2.70, 95\% CI: 1.06-6.86), hypertension (OR: 1.45, 95\% CI: 1.01-2.08), mild CKD (OR: 2.23, 95\% CI: 1.46-3.40), moderate CKD (OR: 3.39, 95\% CI: 2.05-5.62), severe CKD (OR: 6.95, 95\% CI: 3.48-13.90), AMI (OR: 3.24, 95\% CI: $2.29-4.58$ ) and age > 75 years (OR: $2.02,95 \% \mathrm{CI}$ : 1.41-2.88) were independently associated with CI-AKI (Table 2).

\section{PAR of risk factors of $\mathrm{Cl}-\mathrm{AKI}$}

Among the four risk factors of interest of CI-AKI, the prevalence was lowest for hypotension $(2.58 \%)$ and highest for HF symptoms (54.85\%). The PAR was highest for HF symptoms (38.06, 95\% CI: 20.15-53.05\%), followed by hypoalbuminemia $(17.69,95 \% \mathrm{CI}: 1.33-33.60 \%)$ and high contrast volume (12.91, 95\% CI: 3.34-23.46\%), and it was the lowest for hypotension (4.21, 95\% CI: 0.1513.15\%) (Fig. 2a).

As for the other risk factors that were not modifiable, the PAR of AMI was $45.88 \%$ (95\% CI: 32.81-57.53\%), and it was $20.38 \%$ (95\% CI: $0.57-38.06 \%$ ) for hypertension, $14.44 \%$ (95\% CI: $6.35-23.73 \%$ ) for age $>75,12.40 \%$ (95\% CI: $5.03-21.64 \%$ ) for mild CKD, $10.92 \%$ (95\% CI: 5.11-19.16\%) for moderate CKD, $10.63 \%$ (95\% CI: $4.73-$ 20.51\%) for severe CKD (Fig. 2b).

\section{Discussion}

Our study was the first one to estimate the proportion of CI-AKI attributed to four risk factors (HF symptoms, hypoalbuminemia, hypotension, and high contrast volume) that are commonly documented in cardiovascular patients and are potentially modifiable with populationlevel changes in operation strategy and pharmacological therapy. The highest PAR was found for HF symptoms, followed by hypoalbuminemia, high contrast volume and hypotension.

In our cohort, the incidence of CI-AKI was 7.19\%, which was similar to the previous result regarding patients undergoing selected or emergent procedures [9]. In our analyses, we also found that patients with CI-AKI had a higher 10-year mortality than those without CIAKI, which was a further exploration of previous results [3]. This finding highlights the necessity for new strategies to control several potentially modifiable risk factors, as eliminating these risk factors may cause a great reduction in the incidence of CI-AKI.

Our results indicated that HF symptoms was associated with $38.06 \%$ of the CI-AKI cases, which was the highest among the four modifiable risk factors. Based on this finding, physicians may like to find out whether 
Table 1 Baseline characteristics of patients with or without contrast-induced acute kidney injury

\begin{tabular}{|c|c|c|c|c|c|}
\hline Variables & $\begin{array}{l}\text { Total } \\
(n=3450)\end{array}$ & No. (\%) of patients with available data & $\begin{array}{l}\text { Cl-AKI group } \\
(n=248)\end{array}$ & $\begin{array}{l}\text { Non-Cl-AKl group } \\
(n=3202)\end{array}$ & $P$ value \\
\hline Age, y & $62.94 \pm 11.13$ & $3450(100)$ & $69.11 \pm 11.13$ & $62.45 \pm 10.99$ & $<0.001$ \\
\hline Age $>75, n(\%)$ & $571(16.55)$ & $3450(100)$ & $89(35.89)$ & $482(15.05)$ & $<0.001$ \\
\hline Female sex, n (\%) & $802(23.25)$ & $3450(100)$ & $67(27.02)$ & $735(22.95)$ & 0.145 \\
\hline Weight, kg & $64.89 \pm 10.75$ & $3419(99.10)$ & $62.38 \pm 10.71$ & $65.07 \pm 10.73$ & $<0.001$ \\
\hline $\mathrm{SBP}, \mathrm{mmHg}$ & $128.85 \pm 20.48$ & $3439(99.68)$ & $127.78 \pm 25.55$ & $128.93 \pm 20.04$ & 0.492 \\
\hline $\mathrm{DBP}, \mathrm{mmHg}$ & $75.95 \pm 11.91$ & $3438(99.65)$ & $74.74 \pm 12.74$ & $76.04 \pm 11.84$ & 0.123 \\
\hline $\mathrm{HR}$, bpm & $75.07 \pm 13.42$ & $3437(99.62)$ & $79.81 \pm 17.07$ & $74.71 \pm 13.03$ & $<0.001$ \\
\hline \multicolumn{6}{|l|}{ Medical history } \\
\hline$C A D, n(\%)$ & $3077(89.76)$ & $3428(99.36)$ & $234(95.12)$ & $2843(89.34)$ & 0.004 \\
\hline Chronic heart failure, n (\%) & $1961(56.99)$ & $3441(99.74)$ & $182(73.39)$ & $1779(55.72)$ & $<0.001$ \\
\hline CKD, n (\%) & $643(18.64)$ & $3450(100)$ & $114(45.97)$ & $529(16.52)$ & $<0.001$ \\
\hline CKD stages & & & & & $<0.001$ \\
\hline Mild CKD, n (\%) & $397(11.51)$ & & $50(20.16)$ & $347(10.84)$ & \\
\hline Moderate CKD, n (\%) & $177(5.13)$ & & $35(14.11)$ & $142(4.43)$ & \\
\hline Severe CKD, n (\%) & $69(2.00)$ & & $29(11.69)$ & $40(1.25)$ & \\
\hline Hypotension, n (\%) & $89(2.58)$ & $3445(99.86)$ & $28(11.43)$ & $61(1.91)$ & $<0.001$ \\
\hline LVEF, \% & $57.78 \pm 12.26$ & 3008 (87.19) & $51.17 \pm 12.90$ & $58.32 \pm 12.05$ & $<0.001$ \\
\hline LVEF < 40\%, n (\%) & $294(9.77)$ & 3008 (87.19) & $43(18.94)$ & $251(9.03)$ & $<0.001$ \\
\hline HF symptoms, n (\%) & $1876(54.85)$ & $3420(99.13)$ & $170(68.55)$ & $1706(53.78)$ & $<0.001$ \\
\hline Hypertension, n (\%) & $1962(56.89)$ & $3449(99.97)$ & $172(69.35)$ & $1790(55.92)$ & $<0.001$ \\
\hline Hyperlipidemia, n (\%) & $507(14.70)$ & $3450(100)$ & $27(10.89)$ & $480(14.99)$ & 0.079 \\
\hline Smoking, n (\%) & $1371(39.74)$ & $3450(100)$ & $89(35.89)$ & $1282(40.04)$ & 0.198 \\
\hline Hypoalbuminemia, n (\%) & $1334(44.78)$ & $2979(86.35)$ & $126(69.61)$ & $1208(43.17)$ & $<0.001$ \\
\hline Anemia, n (\%) & $1086(31.81)$ & $3414(98.96)$ & $116(47.15)$ & $970(30.62)$ & $<0.001$ \\
\hline AMI, n (\%) & $1300(37.85)$ & $3435(99.57)$ & $163(65.99)$ & $1137(35.66)$ & $<0.001$ \\
\hline Diabetes, n (\%) & $817(23.69)$ & $3448(99.94)$ & $71(28.63)$ & $746(23.31)$ & 0.058 \\
\hline \multicolumn{6}{|l|}{ Laboratory measurements } \\
\hline $\mathrm{LDL}-\mathrm{C}, \mathrm{mmol} / \mathrm{L}$ & $2.74 \pm 0.97$ & $2874(83.30)$ & $2.97 \pm 1.01$ & $2.72 \pm 0.97$ & 0.001 \\
\hline $\mathrm{HDL}-\mathrm{C}, \mathrm{mmol} / \mathrm{L}$ & $1.00 \pm 1.30$ & $2872(83.25)$ & $0.99 \pm 0.27$ & $1.00 \pm 1.34$ & 0.682 \\
\hline NTpro-BNP, pg/mL & $1374.48 \pm 3404.48$ & $2326(67.42)$ & $5442.54 \pm 8475.37$ & $1092.05 \pm 2492.00$ & $<0.001$ \\
\hline $\mathrm{Hs}-\mathrm{CRP}, \mathrm{mg} / \mathrm{L}$ & $17.49 \pm 33.91$ & $2644(76.64)$ & $42.16 \pm 50.94$ & $15.57 \pm 31.42$ & $<0.001$ \\
\hline Lpa, mg/dL & $30.68 \pm 34.43$ & $3037(88.03)$ & $31.28 \pm 33.54$ & $30.63 \pm 34.50$ & 0.785 \\
\hline $\mathrm{SCR}, \mu \mathrm{mol} / \mathrm{L}$ & $92.35 \pm 42.37$ & $3450(100)$ & $117.14 \pm 58.53$ & $90.42 \pm 40.23$ & $<0.001$ \\
\hline eGFR, $\mathrm{mL} / \mathrm{min} / 1.73 \mathrm{~mm}^{2}$ & $80.95 \pm 25.55$ & $3450(100)$ & $67.81 \pm 36.71$ & $81.97 \pm 24.18$ & $<0.001$ \\
\hline Serum urea nitrogen, mg/dL & $5.29 \pm 2.55$ & $3414(98.96)$ & $6.85 \pm 3.73$ & $5.17 \pm 2.40$ & $<0.001$ \\
\hline Hemoglobin, g/L & $132.98 \pm 16.35$ & $3072(89.04)$ & $124.88 \pm 20.52$ & $133.52 \pm 15.89$ & $<0.001$ \\
\hline $\mathrm{HbA} 1 \mathrm{c}, \%$ & $6.54 \pm 1.32$ & $2648(76.75)$ & $6.78 \pm 1.50$ & $6.52 \pm 1.31$ & 0.024 \\
\hline Serum albumin, g/L & $35.23 \pm 7.04$ & $2979(86.35)$ & $32.34 \pm 4.58$ & $35.42 \pm 7.13$ & $<0.001$ \\
\hline \multicolumn{6}{|l|}{ Medications } \\
\hline ACEI/ARB, n (\%) & $3019(87.53)$ & $3449(99.97)$ & $201(81.38)$ & $2818(88.01)$ & 0.002 \\
\hline Beta blocker, n (\%) & $2919(84.63)$ & $3449(99.97)$ & $176(70.97)$ & $2743(85.69)$ & $<0.001$ \\
\hline Statin, n (\%) & $3314(96.09)$ & 3449 (99.97) & $233(94.33)$ & $3081(96.22)$ & 0.140 \\
\hline Diuretics, n (\%) & $654(18.96)$ & 3449 (99.97) & $118(47.58)$ & $536(16.74)$ & $<0.001$ \\
\hline
\end{tabular}


Table 1 Baseline characteristics of patients with or without contrast-induced acute kidney injury (Continued)

\begin{tabular}{|c|c|c|c|c|c|}
\hline Variables & $\begin{array}{l}\text { Total } \\
(n=3450)\end{array}$ & No. (\%) of patients with available data & $\begin{array}{l}\text { Cl-AKI group } \\
(n=248)\end{array}$ & $\begin{array}{l}\text { Non-Cl-AKI group } \\
(n=3202)\end{array}$ & $P$ value \\
\hline Antibiotic, n (\%) & $527(17.11)$ & $3080(89.28)$ & $73(39.25)$ & $454(15.69)$ & $<0.001$ \\
\hline $\mathrm{CCB}, \mathrm{n}(\%)$ & $594(17.26)$ & $3442(99.77)$ & $37(15.10)$ & $557(17.42)$ & 0.354 \\
\hline PPI, n (\%) & $1456(42.30)$ & $3442(99.77)$ & $154(62.60)$ & $1302(40.74)$ & $<0.001$ \\
\hline Metformin, n (\%) & $81(2.63)$ & $3079(89.25)$ & $2(1.08)$ & $79(2.73)$ & 0.236 \\
\hline \multicolumn{6}{|l|}{ Procedure } \\
\hline $\mathrm{PCl}, \mathrm{n}(\%)$ & $2087(67.65)$ & $3085(89.42)$ & $143(76.06)$ & $1944(67.10)$ & 0.011 \\
\hline Contrast volume, $\mathrm{mL}$ & $126.53 \pm 64.54$ & $3447(99.91)$ & $135.44 \pm 64.34$ & $125.84 \pm 66.50$ & $<0.001$ \\
\hline Contrast volume $>155, \mathrm{n}(\%)$ & $852(24.72)$ & $3447(99.91)$ & $80(32.26)$ & $772(24.13)$ & 0.004 \\
\hline Peri-procedure IABP, n (\%) & $133(3.86)$ & $3450(100)$ & $57(22.98)$ & $76(2.37)$ & $<0.001$ \\
\hline
\end{tabular}

Abbreviations: $C I-A K I$ contrast-induced acute kidney injury, SBP systolic blood pressure, DBP diastolic blood pressure, $H R$ heart rate, $L V E F$ left ventricular ejection fraction, $H F$ heart failure, $C K D$ chronic kidney disease, $A M I$ acute myocardial infarction, $L D L-C$ low-density lipoprotein-C, $H D L-C$ high-density lipoprotein-C, $H S$ - $C R P$ high-sensitivity C-reactive protein, $S C R$ serum creatinine, Lpa lipoprotein a, eGFR estimated glomerular filtration rate, $A C E I$ angiotensin-converting enzyme inhibitor, $A R B$ angiotensin-receptor blockers, $P P I$ proton pump inhibitors, $C C B$ calcium channel blocker, $P C I$ percutaneous coronary intervention, $C A D$ coronary artery disease, $I A B P$ intra-aortic balloon pump

heart function improving interventions before the procedure, such as dopamine and recombinant human brain natriuretic peptide (rhBNP), may help reducing the incidence of CI-AKI. In a placebo-controlled, randomized trial, Zhang et al. [14] assigned 149 acute myocardial infarction patients with HF symptoms undergoing emergency PCI to receive rhBNP or placebo. They found that periprocedural use of rhBNP could further promote the recovery of renal function and decrease the occurrence of CI-AKI. Further large, high-quality studies regarding heart function improving interventions to prevent worsen renal function are warranted.

Our study indicated that hypoalbuminemia had the second highest PAR for CI-AKI, which was somewhat unexpected. One possible explanation for our findings is that the patients in our cohort have a relatively high prevalence of hypoalbuminemia (44.78\%), whereas it has also been reported by some previous studies, especially in patients with HF symptoms $[15,16]$. In our study, more than half of the patients had HF symptoms. In addition, a previous study reported that the decline in albumin level appears to be caused by malnutrition and health-related factors with lower household incomes, which may be another reason for the high proportion of hypoalbuminemia in patients with cardiovascular disease in developing countries $[17,18]$. The association between hypoalbuminemia and CI-AKI has been reported by some studies $[19,20]$. Pooled analysis demonstrated that patients with hypoalbuminemia exhibited a higher CI-AKI rate $(\mathrm{OR}=3.09,95 \% \mathrm{CI}=1.44-6.64, P=0.004)$

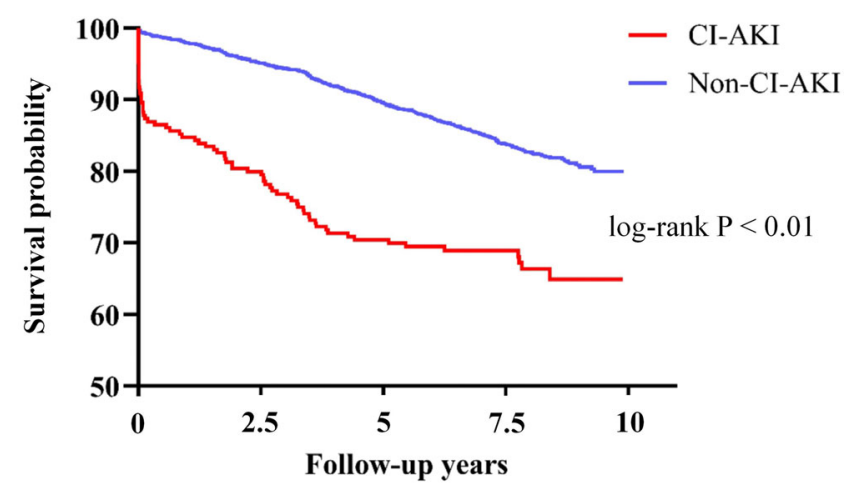

No. at risk

$\begin{array}{lccccc}\text { Non-CI-AKI } & 3130 & 2898 & 2612 & 897 & 0 \\ \text { CI-AKI } & 235 & 171 & 146 & 57 & 0 \\ \text { Follow-up years } & 0 & 2.5 & 5 & 7.5 & 10\end{array}$

Fig. 1 Association between contrast-induced acute kidney injury and long-term survival in patients 'undergoing coronary angiography. Abbreviations: Cl-AKl: contrast-induced acute kidney injury 
Table 2 Univariable and multivariable logistic regression for risk factors of contrast-induced acute kidney injury

\begin{tabular}{|c|c|c|c|c|}
\hline \multirow[b]{2}{*}{ Variables } & \multicolumn{2}{|l|}{ Univariable } & \multicolumn{2}{|l|}{ Multivariable } \\
\hline & $\mathrm{OR}(95 \% \mathrm{Cl})$ & $P$ value & $\mathrm{OR}(95 \% \mathrm{Cl})$ & $P$ value \\
\hline Hypoalbuminemia & $3.02(2.18-4.18)$ & $<0.001$ & $1.48(1.03-2.13)$ & 0.033 \\
\hline HF symptoms & $1.87(1.42-2.47)$ & $<0.001$ & $2.12(1.46-3.06)$ & $<0.001$ \\
\hline High contrast volume & $1.50(1.13-1.98)$ & 0.005 & $1.60(1.14-2.24)$ & 0.006 \\
\hline Hypotension & $6.64(4.16-10.60)$ & $<0.001$ & $2.70(1.06-6.86)$ & 0.037 \\
\hline Age $>75$ years & $3.16(2.40-4.17)$ & $<0.001$ & $2.02(1.41-2.88)$ & $<0.001$ \\
\hline Weight, kg & $0.98(0.96-0.99)$ & $<0.001$ & & \\
\hline Serum urea nitrogen, $\mathrm{mg} / \mathrm{dL}$ & $1.17(1.13-1.21)$ & $<0.001$ & & \\
\hline Hypertension & $1.78(1.35-2.36)$ & $<0.001$ & $1.45(1.01-2.08)$ & 0.042 \\
\hline Anemia & $2.02(1.56-2.63)$ & $<0.001$ & & \\
\hline Coronary artery disease & $2.33(1.29-4.20)$ & 0.005 & & \\
\hline Mild CKD vs non-CKD & $2.87(2.04-4.05)$ & $<0.001$ & $2.23(1.46-3.40)$ & $<0.001$ \\
\hline Moderate CKD vs non-CKD & $4.92(3.27-7.40)$ & $<0.001$ & $3.39(2.05-5.62)$ & $<0.001$ \\
\hline Severe CKD vs non-CKD & $14.46(8.70-24.05)$ & $<0.001$ & $6.95(3.48-13.90)$ & $<0.001$ \\
\hline LVEF $<40$ & $2.36(1.65-3.36)$ & $<0.001$ & & \\
\hline Acute myocardial infarction & $3.50(2.66-4.60)$ & $<0.001$ & $3.24(2.29-4.58)$ & $<0.001$ \\
\hline $\mathrm{PCl}$ & $1.56(1.10-2.20)$ & 0.012 & & \\
\hline
\end{tabular}

Abbreviations: $O R$ odds ratio, $H F$ heart failure, $C K D$ chronic kidney disease, $L V E F$ left ventricular ejection fraction, $P C I$ Percutaneous coronary intervention

[21]. Our finding of hypoalbuminemia as an independent risk factor for CI-AKI was consistent with these studies. Although the mechanism of hypoalbuminemia in the occurrence and development of CI-AKI has not been fully elucidated, possible underlying mechanisms linking CIAKI and hypoalbuminemia may be endothelial dysfunction, oxidative stress and inflammation predisposing to CI-AKI [22-24]. In addition, reducing the incidence of acute kidney injury (AKI) by improving hypoalbuminemia has been proven effective by some studies. In a randomized, double-blind trial, Lee et al. [25] found that patients with hypoalbuminemia

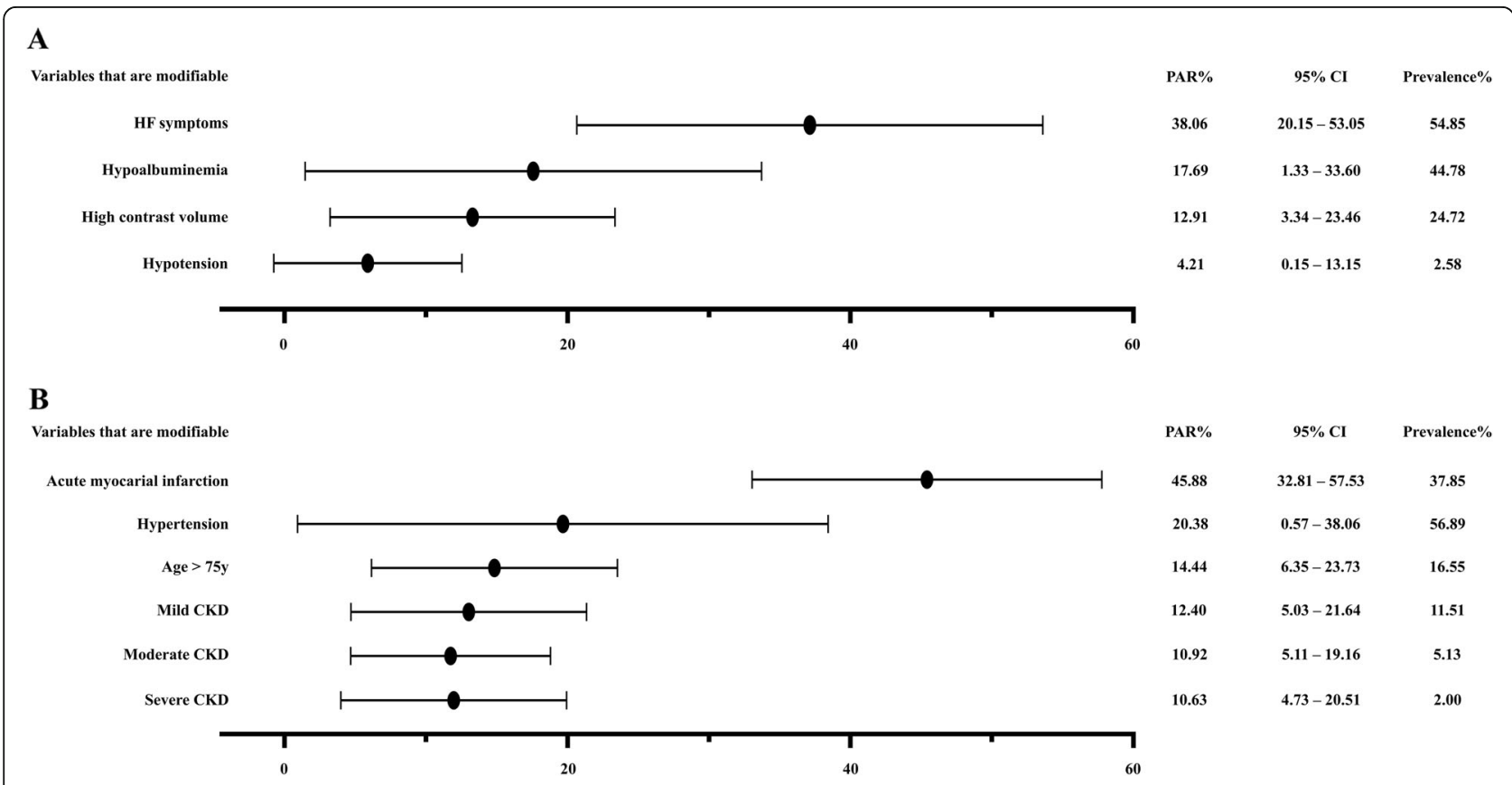

Fig. 2 a: Population attributable risks of the risk factors of interest; b: Population attributable risks of the unmodifiable risk factors. Abbreviations: PAR: population attributable risk; HF: heart failure; CKD: chronic kidney disease 
administered 20\% human albumin before the operation demonstrated a lower incidence of postoperative AKI than those administered an equal volume of saline (13.7\% vs. $25.7 \%$; RR $(95 \% \mathrm{CI})=0.533(0.296-0.961) ; P=$ 0. 048). Our findings suggest that a substantial proportion (17.69\%) of CI-AKI can be attributed to hypoalbuminemia alone, indicating that interventions that improve hypoalbuminemia have the potential to eliminate a large proportion of CI-AKI in patients undergoing CAG.

Our research also showed that high contrast volume and hypotension were independent risk factors of CIAKI, which was consistent with previous studies $[1,11]$ and can explain 12.91 and $4.21 \%$ of CI-AKI cases, respectively. The third-place ranking of high contrast volume among the four risk factors of interest was not unexpected. Recent evidence from cohort studies and meta-analyses demonstrates that we may overestimate the CI-AKI risk conferred by exposure to contrast agents. A meta-analysis involving 25,950 patients who had intravenous administration of iodinated contrast material showed that the risk of CI-AKI was not significantly associated with contrast exposure (RR 0.79, 95\% CI $0.62-1.02 ; P>0.05$ ) [26]. Even in critically ill patients, iodinated contrast medium exposure does not significantly increase the incidence of AKI [27]. The OR of hypotension was the highest among the four modifiable risk factors, while its prevalence was the lowest (2.58\%), which made its PAR rank last among all risk factors of interest. The prevalence of hypotension in our cohort was lower than previous results [11], which may be due to the differences between patients.

Our study had some limitations. First, it was an ancillary study of an observational cohort conducted in a single center located in south China, so the prevalence of the risk factors may not be representative enough. However, this is one of the largest prospective CI-AKI cohorts, and PAR can only be calculated based on observational data. Moreover, our sample included patients at various levels of risk of CI-AKI, which made the results more generalizable. Second, risk factors of interest in this study could not cover all known and unknown CI-AKI risk factors, though the screening process was based on statistical methods, clinical importance and the results of previous high-quality studies. Third, since this was an observational study, we can only suggest that modifying these risk factors may lead to a lower incidence of CI-AKI rather than prove it. The value our results add is in providing these cost-effective targets for further intervention trials. Finally, our definition of CIAKI may be less popular than others, though its association with long-term mortality had been proven by multivariable Cox regression.

\section{Conclusions}

The four risk factors of interest (e.g., HF symptoms, hypoalbuminemia) could be important and cost-effective targets for prevention and treatment strategies to reduce the risk of CI-AKI. Future exploration of CI-AKI prevention strategies targeting these modifiable risk factors is warranted. In addition, our results must be interpreted with caution, as the prevalence of these risk factors may be different between regions. Larger numbers would be needed to draw more definite conclusions and apply the findings to clinical practice.

\section{Supplementary information}

Supplementary information accompanies this paper at https://doi.org/10. 1186/s12872-020-01570-6.

Additional file 1. Selection of the definition of contrast-induced acute kidney injury.

Additional file 2. Landmark analysis discriminating between all-cause mortality before and after 90-days of follow-up.

\section{Abbreviations}

Cl-AKI: Contrast-induced acute kidney injury; MARCE: Major adverse renal and cardiovascular events; ESC: European society of cardiology; EACTS: European association for cardio-thoracic surgery; RRTs: Renal replacement therapies; PAR: Population attributable risk; CAG: Coronary angiography;

PCl: Percutaneous coronary intervention; HF: Heart failure; Scr: Serum creatinine; ALB: Serum albumin; ORs: Odds ratios; CAD: Coronary artery disease; AMI: Acute myocardial infarction; AKI: Acute kidney injury; rhBNP: Recombinant human brain natriuretic peptide

\section{Acknowledgements \\ None.}

\section{Authors' contributions}

Conception and design: All authors; Administrative support: JC; Provision of study materials or patients: All authors; Collection and assembly of data: All authors; Data analysis and interpretation: LL, SC, YL; Manuscript writing and critical revision: All authors; Final approval of manuscript: All authors;

Obtained funding: YL, JC.

\section{Funding}

This work was supported by the Beijing Lisheng Cardiovascular Pilot Foundation [grant no. LHJJ201612127], the "Lixin Yangfan" Optimized Antithrombus Research Fund [grant no. BJUHFCSOARF201801-10], the Progress in Science and Technology Project of Guangzhou [grant no. 201904010470], the Access Research Fund [grant no. 2018-CCA-AF-037], and the China Youth Clinical Research Fund [grant no. 2017-CCA-VG-020]. The funding body did not participate in the design of the study and collection, analysis, and interpretation of data and in writing the manuscript.

\section{Availability of data and materials}

The datasets generated and analysed during the current study are not publicly available due the institution policy but are available from the corresponding author on reasonable request.

\section{Ethics approval and consent to participate}

The study was approved by the Ethics Committee of Guangdong Provincial People's Hospital. All the patients included in this study signed written informed consent.

\section{Consent for publication}

Not applicable.

Competing interests

The authors declare that they have no competing interests. 


\section{Author details}

The Second School of Clinical Medicine, Southern Medical University, Guangzhou 510515, Guangdong, China. 'Department of Cardiology, Provincial Key Laboratory of Coronary Heart Disease, Guangdong Cardiovascular Institute, Guangdong Provincial People's Hospital, Affiliated Guangdong Provincial People's Hospital of South China University of Technology, Guangdong Academy of Medical Sciences, Guangzhou 510080, Guangdong, China. ${ }^{3}$ Department of Cardiology, the People's Hospital of Guangxi Zhuang Autonomous Region, Nanning, Guangxi, China. ${ }^{4}$ Guangdong Provincial People's Hospital, School of Medicine, South China University of Technology, Guangzhou, Guangdong, China. ${ }^{5}$ Department of Emergency and Critical Care Medicine, Guangdong Provincial People's Hospital and Guangdong Academy of Medical Sciences, Guangzhou, Guangdong, China. ${ }^{6}$ Department of Cardiology, Longyan First Affiliated Hospital of Fujian Medical University, Longyan 364000, Fujian, China. ${ }^{7}$ School of Pharmacy, Guangdong Pharmaceutical University, Guangzhou, Guangdong, China.

Received: 5 March 2020 Accepted: 3 June 2020

Published online: 12 June 2020

\section{References}

1. Allen D, Ma B, Leung K, Graham M, Pannu N, Traboulsi M, Goodhart D, Knudtson M, James M. Risk prediction models for contrast-induced acute kidney injury accompanying cardiac catheterization: systematic review and meta-analysis. Can J Cardiol. 2017:33(6):724-36.

2. Mehran R, Dangas GD, Weisbord SD. Contrast-associated acute kidney injury. N Engl J Med. 2019;380(22):2146-55.

3. Yang Y, George KC, Luo R, Cheng Y, Shang W, Ge S, Xu G. Contrast-induced acute kidney injury and adverse clinical outcomes risk in acute coronary syndrome patients undergoing percutaneous coronary intervention: a metaanalysis. BMC Nephrol. 2018;19(1):374.

4. Neumann FJ, Sousa-Uva M, Ahlsson A, Alfonso F, Banning AP, Benedetto U, Byrne RA, Collet JP, Falk V, Head SJ, et al. 2018 ESC/EACTS guidelines on myocardial revascularization. Eur Heart J. 2019;40(2):87-165.

5. Spiegelman D, Hertzmark E, Wand HC. Point and interval estimates of partial population attributable risks in cohort studies: examples and software. Cancer Causes Control. 2007;18(5):571-9.

6. Liu Y, Liang X, Xin S, Liu J, Sun G, Chen S, Cen X, Dai X, He Y, Song F, et al. Risk factors for contrast-induced acute kidney injury (Cl-AKI): protocol for systematic review and meta-analysis. BMJ Open. 2019;9(8):e030048.

7. Liu Y, Chen J, Tan N, Zhou Y, Yu D, Chen Z, He Y, Liu Y, Luo J, Huang W, et al. Safe limits of contrast vary with hydration volume for prevention of contrast-induced nephropathy after coronary angiography among patients with a relatively low risk of contrast-induced nephropathy. Circ Cardiovasc Interv. 2015;8(6):e001859.

8. Jiang W, Yu J, Xu J, Shen B, Wang Y, Luo Z, Wang C, Ding X, Teng J. Impact of cardiac catheterization timing and contrast media dose on acute kidney injury after cardiac surgery. BMC Cardiovasc Disord. 2018;18(1):191.

9. Tsai TT, Patel UD, Chang TI, Kennedy KF, Masoudi FA, Matheny ME, Kosiborod M, Amin AP, Messenger JC, Rumsfeld JS, et al. Contemporary incidence, predictors, and outcomes of acute kidney injury in patients undergoing percutaneous coronary interventions: insights from the NCDR Cath-PCI registry. JACC Cardiovasc Interv. 2014;7(1):1-9.

10. Chi G, Gibson CM, Liu Y, Hernandez AF, Hull RD, Cohen AT, Harrington RA Goldhaber SZ. Inverse relationship of serum albumin to the risk of venous thromboembolism among acutely ill hospitalized patients: analysis from the APEX trial. Am J Hematol. 2019;94(1):21-8.

11. Mehran R, Aymong ED, Nikolsky E, Lasic Z, lakovou I, Fahy M, Mintz GS, Lansky AJ, Moses JW, Stone GW, et al. A simple risk score for prediction of contrast-induced nephropathy after percutaneous coronary intervention: development and initial validation. J Am Coll Cardiol. 2004;44(7):1393-9.

12. Wright RS, Anderson JL, Adams CD, Bridges CR, Casey DE, Ettinger SM, Fesmire FM, Ganiats TG, Jneid H, Lincoff AM, et al. 2011 ACCF/AHA focused update of the guidelines for the management of patients with unstable angina/non-ST-elevation myocardial infarction (updating the 2007 guideline): a report of the American college of cardiology foundation/ American heart association task force on practice guidelines developed in collaboration with the American college of emergency physicians, society for cardiovascular angiography and interventions, and society of thoracic surgeons. J Am College Cardiol. 2011;57(19):1920-59.
13. Willey J, Moon Y, Kahn E, Rodriguez C, Rundek T, Cheung K, Sacco R, Elkind M. Population attributable risks of hypertension and diabetes for cardiovascular disease and stroke in the northern Manhattan study. J Am Heart Assoc. 2014;3(5):e001106.

14. Zhang J, Fu X, Jia X, Fan X, Gu X, Li S, Wu W, Fan W, Su J, Hao G, et al. Btype natriuretic peptide for prevention of contrast-induced nephropathy in patients with heart failure undergoing primary percutaneous coronary intervention. Acta Radiol. 2010;51(6):641-8.

15. Arques $S$, Ambrosi P. Human serum albumin in the clinical syndrome of heart failure. J Card Fail. 2011;17(6):451-8.

16. Ancion A, Allepaerts S, Robinet S, Oury C, Pierard L, Lancellotti P. Serum albumin level and long-term outcome in acute heart failure. Acta Cardiol. 2019;74(6):465-71.

17. India State-Level Disease Burden Initiative Malnutrition Collaborators. The burden of child and maternal malnutrition and trends in its indicators in the states of India: the global burden of disease study 1990-2017. Lancet Child Adolescent Health. 2019;3(12):855-70.

18. Ota A, Kondo N, Murayama N, Tanabe N, Shobugawa Y, Kondo K. Serum albumin levels and economic status in Japanese older adults. PLoS One. 2016;11(6):e0155022

19. Murat SN, Kurtul A, Yarlioglues M. Impact of serum albumin levels on contrast-induced acute kidney injury in patients with acute coronary syndromes treated with percutaneous coronary intervention. Angiology. 2015;66(8):732-7.

20. Hassan K, Fadi H. Is hypoalbuminemia a prognostic risk factor for contrastinduced nephropathy in peritoneal dialysis patients? Ther Clin Risk Manag. 2014;10:787-95.

21. Zhang E, Lu Y, Chen G, Huang L, Zhang J, Wang C, Qin Q. Predictive value of Hepatorenal status in contrast-induced nephropathy among patients receiving coronary angiography and/or intervention: a systematic review and meta-analysis. Angiology. 2019;70(7):633-41.

22. Zhang W, Frei B. Albumin selectively inhibits TNF alpha-induced expression of vascular cell adhesion molecule-1 in human aortic endothelial cells. Cardiovasc Res. 2002;55(4):820-9.

23. Bilasy M, Oraby M, Ismail H, Maklady F. Effectiveness of theophylline in preventing contrast-induced nephropathy after coronary angiographic procedures. J Interv Cardiol. 2012;25(4):404-10.

24. Borekci A, Gur M, Turkoglu C, Cayli M, Selek S, Kaypakli O, Ucar H, Coskun M, Seker T, Koc M, et al. Oxidative stress and paraoxonase 1 activity predict contrast-induced nephropathy in patients with ST-segment elevation myocardial infarction undergoing primary percutaneous coronary intervention. Angiology. 2015;66(4):339-45.

25. Lee E, Kim W, Kim J, Chin J, Choi D, Sim J, Choo S, Chung C, Lee J, Choi I. Effect of exogenous albumin on the incidence of postoperative acute kidney injury in patients undergoing off-pump coronary artery bypass surgery with a preoperative albumin level of less than $4.0 \mathrm{~g} / \mathrm{dl}$. Anesthesiology. 2016;124(5):1001-11.

26. McDonald J, McDonald R, Comin J, Williamson E, Katzberg R, Murad M, Kallmes D. Frequency of acute kidney injury following intravenous contrast medium administration: a systematic review and meta-analysis. Radiology. 2013;267(1):119-28.

27. Ehrmann S, Quartin A, Hobbs B, Robert-Edan V, Cely C, Bell C, Lyons G, Pham T, Schein R, Geng Y, et al. Contrast-associated acute kidney injury in the critically ill: systematic review and Bayesian meta-analysis. Intensive Care Med. 2017:43(6):785-94.

\section{Publisher's Note}

Springer Nature remains neutral with regard to jurisdictional claims in published maps and institutional affiliations. 